\title{
KOSTANT PARTITION FUNCTIONS FOR AFFINE KAC-MOODY ALGEBRAS
}

\author{
T. S. SANTHANAM \\ Department of Science and Mathematics \\ Parks College of Saint Louis University \\ Cahokia, Illinois 62206, USA \\ R. CHAKRABARTI \\ Department of Theoretical Physics \\ University of Madras \\ Madras 600025, INDIA
}

(Received April 17, 1991 and in revised form April 17,1995)

\begin{abstract}
Employing the method of generating functions in conjunction with various numberthoretic identities, we obtain recursion relations for the Kostant partition functions for the affine KacMoody algebras The partition functions for the higher rank algebras are expressed in terms of $\widehat{S U}(2)$ partition functions We derive certain number-theoretic identities using the equivalence of our result with the expressions derived by Kac and Peterson
\end{abstract}

KEY WORDS AND PHRASES. Affine Kac-Moody algebras, Kostant partition functions, weightmultiplicities, positive and simple roots, Diophantine equations, Weyl group, Euler identity, Jacobi's triple and quintuple product identities, Tannery-Molk identity

\section{AMS SUBJECT CLASSIFICATION CODES.}

\section{INTRODUCTION}

The weight multiplicities of the finite dimensional Lie algebras are usually computed using Freudenthal's recursive algorithm or Kostant's formula. For algebras of low rank, the latter works better Kostant's formula for the weight multiplicity $M_{\Lambda}(m)$ of a weight $m$ belonging to the irreducible representation with the highest weight $\Lambda$ is given by

$$
M_{\Lambda}(m)=\sum_{s \in w} \delta_{s} K[s(\Lambda+\rho)-(m+\rho)]
$$

where the sum is over the Weyl group $W$ and $\delta_{s}= \pm 1$ specifying the signature of the permutation The root-space element $\rho$ is defined as

$$
\rho=1 / 2 \sum \alpha
$$

where $\alpha \epsilon$ are positive roots The Kostant's partition function $K(X)$ is defined as the number of ways $X$, an arbitrary element of the positive root space, can be written as a sum over the positive roots with nonnegative integral coefficients, where in general each root is counted as many times as its multiplicity The positive roots in turn can be expanded in the basis of the simple roots with non-negative integral coefficients and the value of $K(X)$ is given by all possible solutions of a set of simultaneous Diophantine equations, the number of equations being the same as the rank of the group

The explicit evaluation for $K(X)$ has been carried out by Tarski [1], Gruber et al [2], Klimyk [3], Gruber et al. [4] and others [5]. One of us (T.S.S ) developed a method [6] using generating function techniques to obtain the number of solutions of these simultaneous Diophantine equations Explicit 
formula for various Lie algebras and recursion relations for the Kostant partition functions for the higher rank Lie algebras in terms of the $S U(2)$ partition functions have been obtained

The Kostant partition formula is also of interest in infinite dimensional Kac-Moody algebras. Kac and Peterson [7] have shown that the Kostant multiplicity formula in Eq (1.1) holds also for Kac-Moody algebras $\widehat{G}$ except that the Weyl group $W$ has a semi-direct product structure

$$
W=\bar{W} \wedge T
$$

where $\bar{W}$ is the finite discrete Weyl group of the finite dimensional Lie algebra $G$ contained in $\widehat{G}$ and $T$ is the infinite translation group acting on the co-root lattice. Kac and Peterson [7] also obtained the Kostant partition function for $S U(2)\left(A_{1}^{(1)}\right), S U(3)\left(A_{2}^{(1)}\right)$ and $A_{2}^{(2)}$ case, where the superscripts in parenthesis refer to the order of the automorphisms (twist).

In the present article we will discuss the computation of Kostant partition functions for the KacMoody algebras by explicitly finding the number of simultaneous Diophantine equations discussed earlier in the context of finite dimensional Lie algebras Much of that program can be translated to the KacMoody case. In particular, the generating functional technique in conjunction with certain number theoretic identities can be fruitfully used to yield a recursive algorithm to generate the Kostant partition function for an arbitrary Kac-Moody algebra.

The number theoretic identities we will repeatedly use are listed below: Euler's identity.

Jacobi's triple product identity:

$$
\phi(q) \equiv \prod_{n=1}^{\infty}\left(1-q^{n}\right)=\sum_{k=-\infty}^{\infty}(-1)^{k} q^{\frac{k}{2}(3 k+1)}
$$

$$
\prod_{n=1}^{\infty}\left(1-x^{n} y^{n}\right)\left(1-x^{n} y^{n-1}\right)\left(1-x^{n-1} y^{n}\right)=\sum_{k=-\infty}^{\infty}(-1)^{k} x^{\frac{1}{2}(k+1)} y^{\frac{1}{2}(k-1)},
$$

and, Jacobi's quintuple product identity:

$$
\begin{gathered}
\prod_{n=1}^{\infty}\left(1-u^{2 n} v^{n}\right)\left(1-u^{2 n-1} v^{n-1}\right)\left(1-u^{2 n-1} v^{n}\right)\left(1-u^{4 n-4} v^{2 n-1}\right)\left(1-u^{4 n} v^{2 n-1}\right) \\
=\sum_{k=-\infty}^{\infty}\left[u^{3 k^{2}-2 k} v^{\frac{1}{2}\left(3 k^{2}+k\right)}-u^{3 k^{2}-4 k+1} v^{\frac{1}{2}\left(3 k^{2}-k\right)}\right]
\end{gathered}
$$

These, and other related identities relate an infinite product structure with an infinite sum, and are of relevance to Kac-Moody algebra because of the infinite number of positive roots of the latter

To illustrate the method we will generally adopt in this article, we will now determine the Kostant partition function for the toy example of Virasoro algebra [8]. The Virasoro generators $L_{n}(n \in Z)$ satisfy the commutation relations

$$
\left[L_{n}, L_{m}\right]=(n-m) L_{n}+{ }_{m}+\frac{c}{12}\left(n^{3}-n\right) \delta_{n+m, 0}
$$

where $c$ denotes the central charge. The Cartan subalgebra is given by $\left(-L_{0}\right)$ and $c$ and the positive roots are given by $L_{n}$ for $n>0$. The root vectors can be read from the commutation relations

$$
\left[c, L_{n}\right]=0, \quad\left[-L_{0}, L_{n}\right]=n L_{n} .
$$

The simple and the positive roots of the algebra and respectively

$$
\delta=(0,1), \quad a_{n}=(0, n)=n \delta \text { for } n>0 .
$$

Kostant's partition function $K\left(n_{0}\right)$ is given by the number of solutions of the Diophantine equation

$$
n_{0}=k_{1}+2 k_{2}+3 k_{3}+\ldots
$$

where $k_{\imath}=$ non-negative integers. 
The generating functional for this partition is given by

$$
\prod_{n=1}^{\infty}\left(1-x^{n}\right)^{-1}=\sum_{n_{0}=0}^{\infty} K\left(n_{0}\right) x^{n_{0}}
$$

and therefore

$$
K\left(n_{0}\right)=P\left(n_{0}\right),
$$

where $P\left(n_{0}\right)$ denotes the unrestricted partition of $n_{0}$ into non-negative integers. Now we may use Euler's identity (4) to rewrite Eq. (1.10) as

$$
\sum_{k=-\infty}^{\infty}(-1)^{k} x^{k(3 k+1)} \sum_{n_{0}=0}^{\infty} K\left(n_{0}\right) x^{n_{0}}=1,
$$

which implies that

$$
\sum_{k=-\infty}^{\infty}(-1)^{k} K\left(n_{0}-\frac{k}{2}(3 k+1)\right)=\delta_{n_{0}, 0}
$$

Equation (1.14) can be used to compute $K\left(n_{0}\right)$ recursively.

Recursive relations, similar to Eq. (1.14) can be obtained for all Kac-Moody groups. In a purely number theoretic context Carlitz [9] obtained similar relations for quantities, which are nothing other than Kostant partition functions for $\widehat{S U}(2)$ Kac-Moody algebra. Subsequently we will refer to these relations as Carlitz recursive relations.

The equivalence of the Kostant partition function by the present generating functional method and that developed by Kac et al. [7] demands a number theoretic identity to be satisfied for each group $\widehat{G}$. In the case of $\widehat{S U}(2)$, the identity is the well-known identity [10]. The necessary identity for the $\widehat{S U}(3)$ example will be established here. Similar generalized T.M identities can be generated by adopting the techniques developed here. Another point to note is that the Kostant partition function for any higher rank group $\widehat{G}$ can be written in terms of the Kostant partition function for $\widehat{S U}(2)$ alone. The reason for this is the fact that the Lie group $G$, contained in $\widehat{G}$, has a triplet $\left(E_{\alpha}, E_{-\alpha}, H_{\alpha}\right)$ satisfying $S U(2)$ Lie algebra for each positive root $E_{\alpha}$ and its corresponding Cartan generator $H_{\alpha}$. In fact, the generating functional for $\widehat{G}$ contains that of the maximal subalgebra contained in $\widehat{G}$.

The plan of the paper is as follows. Section 2 will contain our computation for Kostant partition function for $\widehat{S U}(2)\left(A_{1}^{(1)}\right)$. In Section 3, the partition functions for $\widehat{S U}(3)\left(A_{2}^{(1)}\right)$ will be evaluated. Other (Kac-Moody) rank 3 groups, $\widehat{S p}(4)\left(C_{2}^{(1)}\right)$ and $\widehat{G}_{2}\left(G^{(1)}\right)$ will be discussed in the same spirit in Section 4. The example of $A_{2}^{(2)}$, where we will use the quintuple product identity (6), will be discussed in Section 5 . Finally we will conclude in Section 6.

2. PARTITION FUNCTION FOR $\widehat{S U}(2)$

The Kostant partition function for $\widehat{S U}(2)$ can be computed in many different ways. One of the most elegant methods is formulated by using the recursive relations obtained by Carlitz [9] in the number theoretic context. Alternate expressions obtained by Kac et al. [7] can also be derived in the present procedure of formulating in terms of generating functionals by use of the T.M identity [10]. A third procedure of direct enumeration of the Kostant partition function is also possible by examining the range of possible values of each integer variable in the simultaneous Diophantine equations.

The Cartan matrix for $\widehat{S U}(2)\left(A_{1}^{(1)}\right)$ is

$$
A=\left(\begin{array}{rr}
2 & -2 \\
-2 & 2
\end{array}\right)
$$

and the positive roots of this algebra are given by 


$$
\Delta_{+}=(j-1) a_{0}+j a_{1}, j a_{0}+(j-1) a_{1}, j\left(a_{0}+a_{1}\right), \text { for } j=1,2,3 \ldots,
$$

where $a_{0}$ and $a_{1}$ are two simple roots and are given by

$$
a_{0}=(-\alpha, 0,1), \quad a_{1}=(\alpha, 0,0),
$$

where $\alpha$ is the only simple root for the finite dimensional Lie algebra $S U(2)$. The third set of the positive roots are positive imaginary roots and have to be taken into account in the computation of partition function. The Diophantine equations yielding the Kostant partition functions $K\left(n_{0} a_{0}+n_{1} a_{1}\right)$, subsequently denoted as $K\left(n_{0}, n_{1}\right),\left(n_{0}, n_{1}\right.$, are non-negative integers) are

$$
n_{0}=\sum_{\jmath=1}^{n_{0}} j\left(k_{3 j-1}+k_{3 \jmath}+k_{3 \jmath+1}\right)
$$

and

$$
n_{1}=\sum_{j=2}^{n_{0}-1}(j-1) k_{3 \jmath-1}+\sum_{j=1}^{n_{0}} j k_{3 \jmath}+\sum_{\jmath=0}^{n_{0}}(j+1) k_{3 \jmath+1} .
$$

The upper limits in the sums in Equation (2.4) are given for the case $n_{0}<n_{1}$. The limits for the case $n_{0}>n_{1}$ can be easily written. The variables $k_{j}$ are non-negative integers and the number of solutions of Equation (2.4) give the value of the partition function $K\left(n_{0}, n_{1}\right)$ The generating functional for the partitions is easily seen to be

$$
\begin{aligned}
G(x, y) & =\prod_{n=1}^{\infty}\left(1-x^{n-1} y^{n}\right)^{-1}\left(1-x^{n} y^{n-1}\right)^{-1}\left(1-x^{n} y^{n}\right)^{-1} \\
& =\sum_{n_{0}, n_{1}=0}^{\infty} K\left(n_{0}, n_{1}\right) x^{n_{0}} y^{n_{1}} .
\end{aligned}
$$

The second equality is well-defined in the region $|x|,|y|<1$. Viewed as a partition problem, $K\left(n_{0}, n_{1}\right)$ denotes the number of partitions into, not necessarily distinct, parts $(a, a),(b, b-1)$ and $(c-1, c)$ where $a, b, c=1,2,3, \ldots$. If we now use Jacobi's triple product identity in the form of Eq. (1.5), which turns out to be essentially the "denominator identity", we get

$$
\sum_{\gamma=-\infty}^{\infty}(-1)^{\gamma} x^{\gamma(\gamma+1) / 2} y^{\gamma(\gamma-1) / 2} \sum_{n_{0}, n_{1}=0}^{\infty} K\left(n_{0}, n_{1}\right) x^{n_{0}} y^{n_{1}}=1,
$$

which leads to the remarkable relation of Carlitz [9]

$$
\sum_{\gamma=-\infty}^{\infty}(-1)^{\gamma} K(M-1 / 2 \gamma(\gamma+1), \quad N-1 / 2 \gamma(\gamma-1))=\delta_{M, 0} \delta_{N, 0}
$$

Notice that the summation is over all $\gamma$ satisfying

$$
1 / 2 \gamma(\gamma+1) \leq M, 1 / 2 \gamma(\gamma-1) \leq N .
$$

Equation (2.7) completely determines any $\widehat{S U}(2)$ Kostant partition function $K\left(n_{0}, n_{1}\right)$.

Equation (2.5) allows us to relate $K\left(n_{0}, n_{1}\right)$ to other objects in partition problems. If we define $\Gamma(m, n)$ as the number of partitions of $(m, n)$ into not necessarily distinct parts $(a, a-1),(b-1, b)$ where $a, b=1,2,3, \ldots$; we get the expansion

$$
\sum_{n=1}^{\infty}\left(1-x^{n} y^{n-1}\right)^{-1}\left(1-x^{n-1} y^{n}\right)^{-1}=\sum_{m, n-0}^{\infty} \Gamma(m, n) x^{m} y^{n} .
$$

From Eqs (2.5) and (2.8) we get 


$$
K\left(n_{0}, n_{1}\right)=\sum_{\gamma=0}^{\min \left(n_{0}, n_{1}\right)} P(\gamma) \Gamma\left(n_{0}-\gamma, n_{1}-\gamma\right),
$$

where $P(\gamma)$, introduced earlier, satisfies

$$
\prod_{n=1}^{\infty}\left(1-x^{n}\right)^{-1}=\sum_{\gamma=0}^{\infty} P(\gamma) x^{\gamma}
$$

Also, if we set

$$
\prod_{n=1}^{\infty}\left(1-x^{n} y^{n-1}\right)^{-1}\left(1-x^{n-1} y^{n}\right)^{-1}\left(1-x^{2 n} y^{2 n}\right)^{-1}=\sum_{m, n=0}^{\infty} \pi(m, n) x^{m} y^{n}
$$

so that $\pi(m, n)$ is the number of partitions of $(m, n)$ into not necessarily distinct parts $(a, a-1)$, $(b-1, b)$ and $(2 c, 2 c)$ where $a, b, c=1,2,3 \ldots$, we obtain

$$
K\left(n_{0}, n_{1}\right)=\sum_{\gamma=0}^{\operatorname{mn}\left(n_{0}, n_{1}\right)} Q(\gamma) \pi\left(n_{0}-\gamma, n_{1}-\gamma\right),
$$

where

$$
\prod_{n=1}^{\infty}\left(1+x^{n}\right)=\sum_{y=0}^{\infty} Q(\gamma) x^{\gamma}
$$

Alternately, Eq. (2.5) can be used to derive the expression obtained by Kac and Peterson [7]. For this purpose, we make a change in variable in (2.5) $q=x y$ and re-express $G(x, y)(\equiv \tilde{G}(q, y))$ as

$$
\tilde{G}(q, y)=[\phi(q)]^{-1} \prod_{n=1}^{\infty}\left(1-q^{n} y\right)^{-1}\left(1-q^{n} y^{-1}\right)^{-1} .
$$

To proceed further we use the T.M identity [10], which is

$$
\prod_{n=1}^{\infty}\left(1-q^{n} y\right)^{-1}\left(1-q^{n} y^{-1}\right)^{-1}=\sum_{k=-\infty}^{\infty}(-1)^{k+1}[\phi(q)]^{-2} \frac{q^{\frac{k(k-1)}{2}}}{y-q^{-k}}
$$

This identity can be proved by noting that both sides have the same pole structure in $y$-plane, which allows them to differ only by an analytic function that must vanish as both sides vanish asymptotically To consider the case $n_{0} \leq n_{1}$, we need to take into account only the region of summation $k \geq 0$ in the rhs of Eq. (2.15). Keeping this restriction in mind, we get from Eqs. (2 14) and (2.15)

$$
\begin{aligned}
\tilde{G}(q, y) & =\sum_{k=0}^{\infty}(-1)^{k}[\phi(q)]^{-3} \frac{q^{k(k+1) / 2}}{1-q^{k} y} \\
& =\sum_{k=0}^{\infty}(-1)^{k} \sum_{m=0}^{\infty} p^{(3)}(m) q^{m+k(k+1) / 2}\left[\sum_{\mu=0}^{\infty}\left(q^{k} y\right)^{\mu}\right]
\end{aligned}
$$

where $p^{(d)}(n)$ is defined by

$$
[\phi(q)]^{-d}=\sum_{n=0}^{\infty} p^{(d)}(n) q^{n}
$$

Substituting

$$
\mu=\mu_{1}-n_{0} \quad \text { and } \quad n_{0}=m+k\left(n_{1}-n_{0}\right)+\frac{k(k+1)}{2}
$$


we derive the result

$$
K\left(n_{0}, n_{1}\right)=\sum_{k}(-1)^{k} p^{(3)}\left((k+1) n_{0}-k n_{1}-k(k+1) / 2\right) .
$$

In the case of $n_{0}>n_{1}$, we need to consider the region of summation $k<0$ in the rhs of (215). Equations (2.14) and (2.15) now yield

$$
\begin{aligned}
\tilde{G}(q, y) & =\sum_{k<0}(-1)^{k}[\phi(q)]^{-3} \frac{q^{k(k+1) / 2}}{1-q^{k} y} \\
& =-\sum_{k<0}(-1)^{k} \sum_{m=0}^{\infty} p^{(3)}(m) q^{m+k(k-1) / 2} y^{-1}\left[\sum_{\mu=0}^{\infty} z^{-k} y^{-1}\right]^{\mu} .
\end{aligned}
$$

The substitutions

$$
\mu=n_{0}-n_{1}-1, \quad n_{0}=m+k(k+1) / 2-k\left(n_{0}-n_{1}\right),
$$

reduces Eq. (2.19) to the form

$$
\tilde{G}(q, y)=-\sum_{n_{0}>n_{1}>0} \sum_{k<0}(-1)^{k} p^{(3)}\left((k+1) n_{0}-k n_{1}-k(k+1) / 2\right) q^{n_{0}} y^{n_{1}-n_{0}},
$$

which, from Eq. (2.5) immediately gives

$$
K\left(n_{0}, n_{1}\right)=-\sum_{k<0}(-1)^{k} p^{(3)}\left((k+1) n_{0}-k n_{1}-k(k+1) / 2\right)
$$

for the case $n_{0}<n_{1}$.

There is another more primitive way to determine the Kostant partition function directly from the Diophantine equations. We consider the case of $n_{0} \leq n_{1}$, where the limits of Eq. (2.4) are valid. The case for $n_{0}>n_{1}$ can be developed in a parallel way. The number of solutions of Eq. (2.4) which, by definition, is the Kostant partition function can be expressed in the general case as

$$
\begin{aligned}
K\left(n_{0}, n_{1}\right)= & \sum_{k_{3}=0}^{n_{0}} \sum_{k_{4}=0}^{\min \left(n_{0}-k_{3}\right),\left[\frac{n_{1}-k_{3}}{2}\right]} \ldots \\
& \ldots \sum_{k_{3,-1}=0}^{M_{3,-1}} \sum_{k_{3 j=0}}^{M_{3 j}} \sum_{k_{3,+1}=0}^{M_{3,+1}} \sum_{k_{3 n_{0}+1}=0}^{M_{3 n_{0}+1}} 1,
\end{aligned}
$$

where

$$
\begin{aligned}
M_{3 \jmath-1} & =\min \left(\left[\frac{n_{0}-p(j-1)}{j}\right],\left[\frac{n_{1}-Q(j-1)}{j-1}\right]\right), \\
M_{3 \jmath} & =\min \left(\left[\frac{n_{0}-P(j-1)-j k_{3 \jmath-1}}{j}\right],\left[\frac{n_{1}-q(j-1)-(j-1) k_{3 \jmath-1}}{j}\right]\right), \\
M_{3 \jmath+1} & =\min \left(\left[\frac{n_{0}-P(j-1)-j k_{3 \jmath-1}-j k_{3 \jmath}}{j}\right],\left[\frac{n_{1}-q(j-1)-(j-1) k_{3 \jmath-1}-j k_{3 \jmath}}{j+1}\right]\right), \\
P(j-1) & =\sum_{l=1}^{j-1} l\left[\left(1-\delta_{l, 1}\right) k_{3 l-1}+k_{3 l}+k_{3 l+1}\right], \\
Q(j-1) & =\sum_{l=1}^{j-1}\left[(l-1) k_{3 l-1}+k_{3 l}+(l+1) k_{3 l+1}\right] .
\end{aligned}
$$

This method can be generalized to any arbitrary $\widehat{G}$ and computationally it is quite simple. Notice that for the case $n_{1} \geq 2 n_{0}$, the upper limits of the sums in Eq. (2.22) can be proved to be given by the first entry 
in Eq. (2.23) for $M_{3 \jmath-1}, M_{3 \jmath}$ and $M_{3 \jmath+1}$. The resultant expression for $K\left(n_{0}, n_{1}\right)$ as given by Eq. (2.22) is

$$
K\left(n_{0}, n_{1}>2 n_{0}\right)=P^{(3)}\left(n_{0}\right),
$$

which is the first term for the series obtained in Eq. (2.18)

\section{PARTITION FUNCTION FOR $\widehat{S U}(3)$}

Our discussion for $\widehat{S U}(3)$ will closely parallel that of $\widehat{S U}(2)$. The generating functional for the Kostant partition function can be obtained from the knowledge of all the positive roots. On application of the identity in Eq. (1.5), we can establish a recursive relation for the Kostant partition function. The $\widehat{S U}(3)$ generating functional can also be expressed as a product of the $\widehat{S U}(2)$ generating functionals. This relation can be exploited to express the $\widehat{S U}(3)$ partition function in terms of a linear combination of the products of the $\widehat{S U}(2)$ partition functions. Finally, the equivalence of the generating functional method and that developed by Kac et al. [7] demands some number theoretic identity to be satisfied. In fact, the identical values of the Kostant partition functions may be viewed as a "proof" of this identity. Here we adopt a somewhat anachronistic attitude in that we assume this identity and using it prove the results in Re. [7]. The rationale for this is that an independent proof of the identity may exist.

The Cartan matrix for $\widehat{S U}(3)$ is

$$
A=\left(\begin{array}{rrr}
2 & -1 & -1 \\
-1 & 2 & -1 \\
-1 & -1 & 2
\end{array}\right)
$$

and the system of the positive roots are given by

$$
\Delta_{+}= \begin{cases}(\alpha, 0,0) & \\ ( \pm \alpha, 0, n) & \text { for } n>0 \\ (0,0, n) & \text { for } n>0\end{cases}
$$

where $\alpha$ denotes the positive roots of $\widehat{S U}(3)$. The last set of positive roots are the imaginary positive roots and these occur with multiplicity 2 (in general $r$, where $r$ is the rank of the Lie group $G$ ). This degeneracy is an important distinction with the Lie algebras. The simple roots of $\widehat{S U}(3)$ is given by

$$
a_{0}=\left(-\alpha_{1}-\alpha_{2}, 0,1\right), \quad a_{1}=\left(\alpha_{1}, 0,0\right), \quad a_{2}=\left(\alpha_{2}, 0,0\right)
$$

where $\alpha_{1}$ and $\alpha_{2}$ are the simple roots of the $\widehat{S U}(3)$ Lie algebra. As in the case of $\widehat{S U}(2)$, we can write the Diphantine equations corresponding to the $\widehat{S U}(3)$ partition problem. Instead we directly write the generating functional $G(x, y, z)$ as

$$
\begin{aligned}
G(x, y, z)|| x|,| y|,| z|<|=\phi(x y z) \\
\times \prod_{n=1}^{\infty}\left[\left(1-x^{n} y^{n} z^{n}\right)\left(1-x^{n} z^{n} y^{n-1}\right)\left(1-x^{n-1} z^{n-1} y^{n}\right)\right]^{-1} \\
{\left[\left(1-y^{n} z^{n} x^{n}\right)\left(1-y^{n} z^{n} x^{n-1}\right)\left(1-y^{n-1} z^{n-1} x^{n}\right)\right]^{-1} } \\
\quad\left[\left(1-x^{n} y^{n} z^{n}\right)\left(1-x^{n} y^{n} z^{n-1}\right)\left(1-x^{n-1} y^{n-1} z^{n}\right)\right]^{-1}
\end{aligned}
$$

The Kostant partition function $K\left(n_{0}, n_{1}, n_{2}\right)$, corresponding to the root space element $R=\left(n_{1} a_{1}+n_{1} a_{1}+n_{2} a_{2}\right)$ relates to $G(x, y, z)$ as

$$
G(x, y, z)=\sum_{n_{0}, n_{1}, n_{2}=0}^{\infty} K\left(n_{0}, n_{1}, n_{2}\right) x^{n_{0}} y^{n_{1}} z^{n_{2}} .
$$

Using Eqs (1.4), (1.5) in Eqs. (3.4) and (3.5), we get 


$$
\begin{aligned}
\sum_{N=-\infty}^{\infty}(-1)^{N}(x y z)^{N(3 N+1) / 2}= & {\left[\sum_{n_{0}, n_{1}, n_{2}=0}^{\infty} K\left(n_{0}, n_{1}, n_{2}\right) x^{n_{0}} y^{n_{1}} z^{n_{2}}\right] } \\
& {\left[\sum_{\mu, v, p=-\infty}^{\infty}(-1)^{\mu+v+p}(x z)^{\mu(\mu+1) / 2} y^{\mu(\mu-1) / 2}\right.} \\
& \left.(y z)^{v(v+1) / 2} x^{v(v-1) / 2}(x y)^{p(p+1) / 2} z^{p(p-1) / 2}\right]
\end{aligned}
$$

After the following substitutions

$$
\begin{array}{ll}
M_{p}=\frac{\mu(\mu+1)}{2}+\frac{v(v-1)}{2}+\frac{p(p+1)}{2}, & M_{Q}=\frac{\mu(\mu-1)}{2}+\frac{v(v+1)}{2}+\frac{p(p+1)}{2}, \\
M_{R}=\frac{\mu(\mu+1)}{2}+\frac{v(v+1)}{2}+\frac{p(p-1)}{2}, & E_{N}=\frac{N(3 N+1)}{2}
\end{array}
$$

we get the Carlitz recursive relations

$$
\sum_{\mu, v, p=-\infty}^{\infty}(-1)^{\mu+v+p} K\left(P-M_{P}, Q-M_{Q}, R-M_{R}\right)=(-1)^{N} \delta_{p, E_{N}} \delta_{Q, E_{N}} \delta_{R, E_{N}} .
$$

Now we will express the $\widehat{S U}(3)$ partition function as a linear combination of the products of $\widehat{S U}(2)$ partition function. For this we introduce the variable $q(=x y z)$ and re-express the generating functional $\tilde{G}(q, y, z)(=G(x, y, z))$ as

$$
\begin{aligned}
\tilde{G}(q, y, z)=[\phi(q)]^{-2}[(1-y)(1-z)(1-y z)]^{-1} \prod_{n=1}^{\infty} & {\left[\left(1-q^{n} y\right)\left(1-q^{n} z\right)\left(1-q^{n} y z\right)\right.} \\
& \left.\left(1-q^{n} y^{-1}\right)\left(1-q^{n} z^{-1}\right)\left(1-q^{n} y^{-1} z^{-1}\right)\right]^{-1}
\end{aligned}
$$

A comparison with Eq. (2.14) immediately reveals a factorization of the $\widehat{S U}(3)$ generating functional takes place in terms of the $\widehat{S U}(2)$ generating functionals:

$$
\tilde{G}(q, y, z)=\phi(q) \tilde{G}(q, y) \tilde{G}(q, z) \tilde{G}(q, y z) .
$$

Using Eqs. (14), (2.5), (3.5) and equating the coefficients of the identical powers of the variables $q, y$ and $z$ on both sides of Eq. (3.9) we get

$$
\begin{array}{r}
K(M, N, P)=\sum_{m=-\infty}^{\infty} \sum_{p, q, r=0}^{\infty}(-1)^{m} K(M-p-q-m(3 m+1) / 2, r) \\
K(N-q-r-m(3 m+1) / 2, p) \\
K(P-p-r-m(3 m+1) / 2, q),
\end{array}
$$

where the summation variables satisfy the conditions

$$
p+q+m(3 m+1) / 2 \leq M, \quad q+r+m(3 m+1) / 2 \leq N, \quad p+r+m(3 m+1) / 2 \leq P .
$$

Showing the equivalence of the generating functional method of obtaining Kostant partition function with the result of Ref. [7] demands satisfaction of certain identities, which may be referred to as generalized T.M identity. In fact, the equivalence, which can be checked by calculating $K\left(n_{0}, n_{1}, n_{2}\right)$ in both ways for an arbitrary set of positive integers $\left(n_{0}, n_{1}, n_{2}\right)$ may be considered as a proof of these identities. We will, however, take the opposite viewpoint and assume that independent proofs of these identities may be furnished; thus taking these identities as starting points we will attempt to derive the results in Ref. [7] from the generalized functional point of view In the present article, we only write the 
generalized T.M identity for $\widehat{S U}(3)$ algebra and derive the result for $K\left(n_{0}, n_{1}, n_{2}\right)$ first obtained in Ref [7]

The generalized T.M identity for $\widehat{S U}(3)$ may be written as

$$
\begin{aligned}
{[(1-y)(1-z)(1-y z)]^{-1} \prod_{n=1}^{\infty} } & {\left[\left(1-q^{n} y\right)\left(1-q^{n} z\right)\left(1-q^{n} y z\right)\right.} \\
& \left.\times\left(1-q^{n} y^{-1} z^{-1}\right)\left(1-q^{n} z^{-1}\right)\left(1-q^{n} y^{-1}\right)\right]^{-1} \\
= & \text { RHS },
\end{aligned}
$$

where the right hand side of (3.11) can take one of the following forms

$$
\begin{aligned}
& (\mathrm{RHS})_{1}=[\phi(q)]^{-6} \sum_{k, l>0} q^{l(l+k+1)+k(k+1)}\left[\frac{2 k+l+1}{\left(1-q^{k} y\right)\left(1-q^{l} z\right)}+\frac{q^{k} y}{\left(1-q^{k} y\right)^{2}\left(1-q^{l} z\right)}\right] \\
& -\sum_{k, l \geq 0} q^{(l+1)(k+l+1)+k(k+1)}\left[\frac{2 k+l+2}{\left(q^{-k-1} y\right)\left(q^{l+k+1} z\right)}\right. \\
& \left.+\frac{q^{l+k+1} Z}{\left(1-q^{-k-1} y\right)\left(1-q^{l+k+1} Z\right)^{2}}-\frac{q^{-k-1} y}{\left(1-q^{-k-1} y\right)^{2}\left(1-q^{l+k+1} Z\right)}\right] \text {, } \\
& (\mathrm{RHS})_{2}=[\phi(q)]^{-6} \sum_{k, l \geq 0} q^{l(l+k+1)+k(k+1)}\left[\frac{q^{-k} y^{-1}(2 k+l+1)}{\left(1-q^{-k} y^{-1}\right)\left(1-q^{l} z\right)}-\frac{q^{-k} y^{-1}}{\left(1-q^{-k} y^{-1}\right)^{2}\left(1-q^{l} z\right)}\right] \\
& -\sum_{k, l \geq 0} q^{(l+1)(k+l+1)+k(k+1)}\left[\frac{(2 k+l+2) q^{k+1} y^{-1}}{\left(1-q^{k+1} y^{-1}\right)\left(1-q^{l+k+1} z\right)}\right. \\
& \left.+\frac{q^{l+2 k+2} y^{-1} z}{\left(1-q^{k+1} y^{-1}\right)\left(1-q^{l+k+1} z\right)^{2}}+\frac{q^{k+1} y^{-1}}{\left(1-q^{k+1} y^{-1}\right)^{2}\left(1-q^{k+l+1} z\right)}\right] \text {, } \\
& (\mathrm{RHS})_{3}=[\phi(q)]^{-6} \sum_{k, l \geq 0} q^{l(l+k+1)+k(k+1)}\left[\frac{(2 k+l+1) q^{-l} z^{-1}}{\left(1-q^{k} y\right)\left(1-q^{-l} z^{-1}\right)}+\frac{q^{k-l} y z^{-1}}{\left(1-q^{k} y\right)^{2}\left(1-q^{-l} z^{-1}\right)}\right] \\
& -\sum_{k, l \geq 0} q^{(l+1)(l+k+1)+k(k+1)}\left[\frac{(2 k+l+2) q^{-(k+l+1)} z^{-1}}{\left(1-q^{-k-1} y^{-1}\right)\left(1-q^{-l-k-1} z^{-1}\right)}\right. \\
& \left.-\frac{q^{-2 k-l-2} y z^{-1}}{\left(1-q^{-k-1} y\right)^{2}\left(1-q^{-k-l-1} z^{-1}\right)}-\frac{q^{-k-l-1} z^{-1}}{\left(1-q^{-k-1} y\right)\left(1-q^{-k-l-1} z^{-1}\right)^{2}}\right]
\end{aligned}
$$

and

$$
\begin{aligned}
(\mathrm{RHS})_{4}= & {[\phi(q)]^{-6} \sum_{k, l \geq 0} q^{l(l+k+1)+k(k+1)}\left[\frac{(2 k+l+1) q^{-k-l} y^{-1} z^{-1}}{\left(1-q^{-k} y^{-1}\right)\left(1-q^{-l} z^{-1}\right)}-\frac{\left(q^{-k-l} y^{-1} z^{-1}\right)}{\left(1-q^{-k} y^{-1}\right)^{2}\left(1-q^{-l} z^{-1}\right)}\right] } \\
- & \sum_{k, l \geq 0} q^{(l+1)(k+l+1)+k(k+1)}\left[\frac{(2 k+l+2) q^{-l} y^{-1} z^{-1}}{\left(1-q^{k+1} y^{-1}\right)\left(1-q^{-k-l-1} z^{-1}\right)}\right. \\
& \left.+\frac{q^{-l} y^{-1} z^{-1}}{\left(1-q^{k+1} y^{-1}\right)^{2}\left(1-q^{-l-k-1} z^{-1}\right)}-\frac{q^{-l} y^{-1} z^{-1}}{\left(1-q^{k+1} y^{-1}\right)\left(1-q^{-k-l-1} z^{-1}\right)^{2}}\right]
\end{aligned}
$$

The precise meaning of the identity is the following. If we use Taylor's expansion both sides of Eq. (3 11) as a power series in $(q, y$ and $z)$ or $\left(q, y^{-1}\right.$ and $\left.z\right)$ or $\left(q, y, z^{-1}\right)$, the RHS takes the value in Eq (3.12)/Eq. (3.13)/Eq (3.14)/Eq. (3.15) 
For the determination of the $\widehat{S U}(3)$ Kostant partition function in the region $n_{0}<\min \left(n_{1}, n_{2}\right)$, we need to consider the value of RHS in Eq. (3.11) as given in Eq. (3.12). From Eqs. (3.8),(3.11) and (3.12), we obtain

$$
\tilde{G}(q, y, z)=T_{1}-T_{2}
$$

The term $T_{1}$ may be written as

$$
\begin{aligned}
T_{1} & =[\phi(q)]^{-8} \sum_{k, l \geq 0} q^{k(k+1)+l(l+k+1)}\left[\frac{2 k+l+1}{\left(1-q^{k} y\right)\left(1-q^{l} z\right)}-\frac{q^{k} y}{\left(1-q^{k} y\right)^{2}\left(1-q^{l} z\right)}\right] \\
& =\sum_{k, l \geq 0} \sum_{m>0} P^{(8)}(m) q^{m+k(k+1)+l(l+k+1)}\left[\sum_{\mu, v \geq 0}(\mu+2 k+l+1)\left(q^{k} y\right)^{\mu}\left(q^{l} z\right)^{v}\right] .
\end{aligned}
$$

After the substitutions of

$$
n_{0}=m+k \mu+l v+k(k+1)+l(k+l+1), \quad n_{1}=\mu+n_{0}, \quad n_{2}=v+n_{0},
$$

we get from Eq. (3.17)

$$
\begin{gathered}
T_{1}=\sum_{n_{1}, n_{2} \geq n_{0} \geq 0} \sum_{k, l \geq 0}\left(n_{1}-n_{0}+2 k+l+1\right) P^{(8)}\left((k+l+1) n_{0}-k n_{1}-l n_{2}-l(k+l+1)\right) \\
q^{n_{0}} y^{n_{1}-n_{0}} z^{n_{2}-n_{0}} .
\end{gathered}
$$

The term $T_{2}$ is given by

$$
\begin{aligned}
T_{2}= & {[\phi(q)]^{-8} \sum_{k, l \geq 0} q^{k(k+1)+(l+1)(l+k+1)}\left[\frac{(2 k+l+2)}{\left(1-q^{-k-1} y\right)\left(1-q^{l+k+1}\right)}\right.} \\
& \left.+\frac{q^{k+l+1} z}{\left(1-q^{-k-1} y\right)\left(1-q^{-l-k-1} z\right)^{2}}-\frac{q^{-k-1} y}{\left(1-q^{-k-1} y\right)^{2}\left(1-q^{l+k+1} z\right)}\right] \\
= & \sum_{k, l \geq 0} \sum_{m>0} P^{(8)}(m) q^{m+k(k+1)+(l+1)(k+l+1)} \\
& {\left[\sum_{\mu, v>0}(v-\mu+2 k+l+2)\left(q^{-k-1} y\right)^{\mu}\left(q^{l+k+1} z\right)^{v}\right] . }
\end{aligned}
$$

We make the substitutions

$$
n_{0}=m+(l+1)(k+l+1)+k(k+1)+v(l+k+1)-\mu(k+1), \quad n_{1}=\mu+n_{0}, \quad n_{2}=v+n_{0},
$$

which re-expresses $T_{2}$ as

$$
\begin{aligned}
T_{2}=\sum_{n_{1}, n_{2} \geq 0} & \sum_{n, l \geq 0}\left(n_{2}-n_{1}+2 k+l+2\right) P^{(8)}(l+1) n_{0}+(k+1) n_{1}-(l+k+1) n_{2} \\
& -(l+1)(k+l+1)-k(k+1) q^{n_{0}} y^{n_{1}-n_{0}} z^{n_{1}-n_{0}} .
\end{aligned}
$$

From Eqs (3.5), (3.16), (3.19) and (3.20), we get the $\widehat{S U}(3)$ partition function in the range $0 \leq n_{0} \leq \min \left(n_{1}, n_{2}\right)$

$$
\begin{aligned}
K\left(n_{0}, n_{1}, n_{2}\right)= & \sum_{k, l \geq 0}\left(n_{1}-n_{0}+2 k+l+1\right) P^{(8)}\left((k+l+1) n_{0}-k n_{1}-l n_{2}-(k+l+1)-k(k+1)\right) \\
& -\sum_{k, l \geq 0}\left(n_{2}-n_{1}+2 k+l+2\right) P^{(8)}\left((l+1) n_{0}+(k+1) n_{1}-(l+k+1) n_{2}\right. \\
& -(l+1)(k+l+1)-k(k+1)) .
\end{aligned}
$$

Taking other forms of RHS in Eq. (3.11) we can get the partition functions in other regions. The particular form of the identity we have chosen will always lead to the expression of the partition function 
$K\left(n_{0}, n_{1}, n_{2}\right)$ as given in Eq. (3.21). We repeat, we do not have an independent proof of the identity in Eq. (3.11); and, the identical numerical values of $K\left(n_{0}, n_{1}, n_{2}\right)$ given by our method and in Ref. [7] should properly be viewed as a "proof" of the identity (3.11) - nevertheless an independent proof of Eq. (3.11) may exist, which may be subsequently used to get the result of Ref. [7] from our generating functional method.

IV. PARTITION FUNCTION FOR $S \widehat{P}(4)$ AND $\widehat{G}_{2}$ The program developed so far is adequate for determination of the partition function for all Kac-Moody algebras $G$. To demonstrate this point we consider the other Kac-Moody algebras with rank 3 i.e. $S \widehat{P}(4)\left(C_{2}^{(1)}\right)$ and $\widehat{G}_{2}$. Since we gave a detailed exposition of our method earlier, our future discussions will be brief We directly write the generating functional for $S \widehat{P}(4)$ as

$$
\begin{aligned}
G_{c}(x, y, z)|| x|,| y|,| z \mid<1= & \phi\left(x y^{2} z\right)^{2} \prod_{n=1}^{\infty}\left[\left(1-(x y z)^{n} y^{n}\right)\left(1-(x y z)^{n-1} y^{n}\right)\left(1-(x y z)^{n} y^{n-1}\right)\right] \\
& {\left[\left(1-\left(x y^{2}\right)^{n} z^{n}\right)\left(1-\left(x y^{2}\right)^{n-1} z^{n}\right)\left(1-\left(x y^{2}\right)^{n} z^{n-1}\right)\right]^{-1} } \\
& {\left[\left(1-(x y)^{n}(y z)^{n}\right)\left(1-(x y)^{n-1}(y z)^{n}\right)\left(1-(x y)^{n}(y z)^{n-1}\right)\right]^{-1} } \\
& {\left[\left(1-x^{n}\left(y^{2} z\right)^{n}\right)\left(1-x^{n-1}\left(y^{2} z\right)^{n}\right)\left(1-x^{n}\left(y^{2} z\right)^{n-1}\right]^{-1} .\right.}
\end{aligned}
$$

The partition function $K_{c}\left(n_{0}, n_{1}, n_{2}\right)$ is defined by the expansion

$$
G_{c}(x, y, z)=\sum_{n_{0}, n_{1}, n_{2} \geq 0}^{\infty} K_{c}\left(n_{0}, n_{1}, n_{2}\right) x^{n_{0}} y^{n_{1}} z^{n_{2}} .
$$

As done previously, we derive the Carlitz recursive relation for $\widehat{S P}(4)$ by using Eqs. (1 4), (1 5), (4.1) and (4.2)

$$
\begin{aligned}
\sum_{m_{1}, m_{2}, m_{3}, m_{4}=-\infty}^{\infty}(-1)^{\sum_{i=1}^{4} m_{2}} K_{c}\left(P-M_{p}, Q-M_{Q}, R-M_{R}\right) \\
=\sum_{N_{1}, N_{2}=-\infty}^{\infty}(-1)^{N_{1}+N_{2}} \delta_{P, E_{N}} \delta_{Q / 2, E_{N}} \delta_{R, E_{N}}
\end{aligned}
$$

where

$$
\begin{aligned}
& M_{P}=\frac{1}{2} \sum_{i=1}^{4} m_{\imath}\left(m_{\imath}+1\right), \quad M_{Q}=m_{1}^{2}+\frac{m_{2}}{2}\left(m_{2}+1\right)+m_{3}^{2}+m_{4}\left(m_{4}-1\right), \\
& M_{R}=\frac{m_{1}}{2}\left(m_{1}+1\right)+\frac{m_{2}}{2}\left(m_{2}-1\right)+\frac{m_{3}}{2}\left(m_{3}-1\right)+\frac{m_{4}}{2}\left(m_{4}-1\right), \quad \cdot E_{n}=\frac{1}{2} \sum_{\imath=1}^{2} N_{\imath}\left(3 N_{\imath}+1\right) .
\end{aligned}
$$

The partition function $K_{c}\left(n_{0}, n_{1}, n_{2}\right)$ can be expressed in terms of $\widehat{S U}(2)$ partition function. To this effect, we introduce the variable $q\left(=x y^{2} z\right)$ and write the generating functional $\widehat{G}_{c}(q, y, z)\left(=G_{c}(x, y, z)\right)$ as

$$
\tilde{G}_{c}(q, y, z)=[\phi(q)]^{2} \tilde{G}(q, y) \tilde{G}(q, z) \tilde{G}(q, y z) \tilde{G}\left(q, y^{2} z\right) .
$$

We quote our result for $K_{c}(M, N, P)$.

$$
\begin{aligned}
K_{c}(M, N, P)= & \sum_{\mu_{1}, \mu_{2}=-\infty}^{\infty} \sum_{m, n, p, q, r=0}^{\infty}(-1)^{N(\mu)} K(m, n) \\
& K(M-p-q-m-S(\mu), r), K(N-M-q-r-2 n+m-S(\mu), p), \\
& K(P-p-r-n-S(\mu), q) .
\end{aligned}
$$

The arguments of $K(m, n)$ must be non-negative integers and $N(\mu)$ and $S(\mu)$ are defined as 


$$
N(\mu)=\mu_{1}+\mu_{2}, \quad S(\mu)=\frac{1}{2} \sum_{\imath=1}^{2} \mu_{\imath}\left(3 \mu_{\imath}+1\right) .
$$

Comparing Equations (3.9) and (4.4) we notice that

$$
\tilde{G}_{c}(q, y, z)=\phi(q) \tilde{G}(q, y, z) \tilde{G}\left(q, y^{2} z\right) .
$$

This indicates that the generating functional of $\widehat{S P}(4)$ can be factorized in - apart from factors of Euler function - that of $\widehat{S U}(3)$, which is the maximal subalgebra of the former, and other factors of $\widehat{S U}(2)$ generating functionals The latter originates in the extra roots the algebra has over and above its maximal subalgebra. Exploiting this we write the $\widehat{S P}(4)$ partition function $K_{\mathrm{c}}(M, N, P)$ in terms of the partition functions for $\widehat{S U}(3)$ and $\widehat{S U}(2)$ as

$$
\begin{array}{r}
K_{c}(M, N, P)=\sum_{k=-\infty}^{\infty} \sum_{, q=0}^{\infty}(-1)^{k} K\left(M-p-\frac{k(3 k+1)}{2}, \quad N+p-2 q-\frac{k}{2}(3 k+1),\right. \\
\left.P-q-\frac{k}{2}(3 k+1)\right) K(p, q) .
\end{array}
$$

We can continue the same program for $\widehat{G}_{2}\left(G^{(1)}\right)$. We will just quote the results here. A recursive relation can be established for the $\widehat{G}_{2}$ partition function $K_{G}\left(n_{0}, n_{1}, n_{2}\right)$.

$$
\begin{aligned}
& \sum_{m_{\mathrm{i}}, m_{2}, \ldots, m_{6}=-\infty}^{\infty}(-1)^{\sum_{i=1}^{6} m_{1}} K_{G}\left(P-M_{p}, Q-M_{Q}, R-M_{R}\right) \\
= & \sum_{N_{1}, \ldots, N_{4}=-\infty}^{\infty}(-1)^{\sum_{i=1}^{4} N_{1}} \delta_{P, E_{N}} \delta_{Q / 2, E_{N}} \delta_{R / 3, E_{N}}
\end{aligned}
$$

where

$$
\begin{aligned}
M_{P}= & \frac{1}{2} \sum_{i=1}^{6} m_{\imath}\left(3 m_{\imath}+1\right) \\
M_{Q}= & m_{1}^{2}+m_{2}\left(m_{2}+1\right)+m_{3}^{2}+m_{4}^{2}+m_{5}^{2}+m_{6}\left(m_{6}-1\right), \\
M_{R}= & \frac{3 m_{1}}{2}\left(m_{1}-1\right)+\frac{m_{2}}{2}\left(3 m_{2}+1\right)+\frac{m_{3}}{2}\left(3 m_{3}+1\right) \\
& +\frac{m_{4}}{2}\left(3 m_{4}-1\right)+\frac{3 m_{5}}{2}\left(m_{5}-1\right)+\frac{3 m_{6}}{2}\left(m_{6}-1\right), \\
E_{N}= & \frac{1}{2} \sum_{i=1}^{4} N_{i}\left(3 N_{\imath}+1\right) .
\end{aligned}
$$

The factorization of the partition functions in terms of the $\widehat{S U}(2)$ partition functions reads as follows:

$$
\begin{aligned}
K_{G}(M, N, P)= & \sum_{\mu_{1}, \ldots, \mu_{4}=1-\infty}^{\infty}(-1)^{N(\mu)} \sum_{m_{1}, n_{1}, m_{2}, n_{2}, m_{3}, n_{3}, p, q, r=0}^{\infty} \\
& K\left(m_{1}, n_{1}\right) K\left(m_{2}, n_{2}\right) K\left(m_{3}, n_{3}\right) \\
& K\left(M-S(\mu)-p-q-m_{1}-m_{2}-m_{3}, r\right) \\
& K\left(N-M-S(\mu)-q-r-2 m_{1}-n_{1}-n_{2}+m_{3}-2 n_{3}, p\right) \\
& K\left(P-2 M-S(\mu)-p-r+m_{1}-2 n_{1}+2 m_{2}-3 n_{2}+2 m_{3}-3 n_{3}, q\right),
\end{aligned}
$$

where

$$
\begin{aligned}
N(\mu) & =\sum_{i=1}^{4} \mu_{i}, \\
S(\mu) & =\frac{1}{2} \sum_{i=1}^{4} \mu_{i}\left(3 \mu_{\imath}-1\right) .
\end{aligned}
$$


We can again write the $\widehat{G}_{2}$ partition function in terms of the partition function of $\widehat{S U}(3)$, the maximal subgroup of the former and the other $\widehat{S U}(2)$ partition functions. But we omit it as the point has already been made in the case of $S P(4)$.

\section{PARTITION FUNCTION FOR $A_{2}^{(2)}$}

The Cartan matrix in this case is

$$
A=\left(\begin{array}{rr}
2 & -4 \\
-1 & 2
\end{array}\right)
$$

with the set of the positive roots given by

$$
\Delta_{+}=\left\{\begin{array}{l}
4 n \alpha_{0}+(2 n-1) \alpha_{1}, 4(n-1) \alpha_{0}+(2 n-1) \alpha_{1}, \\
(2 n-1) \alpha_{0}+n \alpha_{1},(2 n-1) \alpha_{0}+(n-1) \alpha_{1}, \\
2 n \alpha_{0}+n \alpha_{1},
\end{array}\right.
$$

where $n=1,2,3, \ldots$ and the pair $\alpha_{0}$ and $\alpha_{1}$ denote the simple roots. The generating functional for this case is therefore

$$
\begin{aligned}
\left.G(u, v)\right|_{|u|,|v|<1}= & \prod_{n=1}^{\infty}\left(1-u^{2 n} v^{n}\right)^{-1}\left(1-u^{2 n-1} v^{n-1}\right)^{-1}\left(1-u^{2 n-1} v^{n}\right)^{-1} \\
& \left(1-u^{4 n-4} v^{2 n-1}\right)^{-1}\left(1-u^{4 n} v^{2 n-1}\right)^{-1} \\
= & \sum_{n_{0, n_{1}=0}}^{\infty} K_{A_{2}^{(2)}}\left(n_{0}, n_{1}\right) u^{n_{0}} v^{n_{1}}
\end{aligned}
$$

We now use the quintuple product identity in Eq. (1.6) to derive the Carlitz-type recursive relation

$$
\begin{aligned}
\sum_{m \in Z} K_{A_{2}^{(2)}}( & \left.n_{0}-3 m^{2}+2 m, n_{1}-\frac{1}{2}\left(3 m^{2}+m\right)\right) \\
& -\sum_{m \in Z} K_{A_{2}^{(2)}}\left(n_{0}-3 m^{2}+4 m-1, n_{1}-\frac{1}{2}\left(3 m^{2}-m\right)\right)=\delta_{n_{0,0}} \delta_{n_{1,0}},
\end{aligned}
$$

where the arguments of $K_{A_{2}^{(2)}}$ must be non-negative. This is to be compared to the formula obtained in Ref. [7]

$$
\begin{aligned}
K_{A_{2}^{(2)}}\left(n_{0, n_{1}}\right)= & \sum_{k \geq 1} P^{(2123)}\left((2 k-1) n_{0}-4(k-1) n_{1}-(k-1)(3 k-1)\right) \\
& -\sum_{k \geq 1} P^{(53)}\left(k n_{0}-(2 k-1) n_{1}-\frac{1}{2} k(3 k-1)\right) .
\end{aligned}
$$

\section{CONCLUSION}

We have discussed the method of computing the Kostant partition functions for Kac-Moody algebras using their generating functionals. We have illustrated this method with several examples. The method consists of first enumerating the positive and simple roots which directly furnishes the generating functional for the Kostant partition function Number theoretic identities, such as, the Euler identity, the Jacobi triple product identity and the quintuple product identity have been used to derive Carlitz-type recursive relations. We have also written the partition functions for the higher groups in terms of the $\widehat{S U}(2)$ partition functions. Kac and Peterson computed the partition functions in some special cases using the Weyl group. In turn, we may use their results to obtain the identities, which may be viewed as a generalization of the T.M identity. The root system (in particular, the positive roots) of the Kac-Moody infinite-dimensional Lie algebras $\widehat{g}$ are expressible as infinitely repeated roots of the finite dimensional Lie algebra $g$ contained in $\widehat{g}$, the different sets related by the imaginary roots. This basic fact underlies the denominator identities and enables one to express the denominator in the character function in terms of Theta functions and modular forms. Expressed in these terms, the various identities (infinite products 
expressed as infinite sums) which have been used are immediate consequences of Macdonald identities [11]).

The formalism developed here may be of relevance to the super Kac-Moody algebras. The Freudenthal formula, and its extension to Kac-Moody algebras assume the existence of an invariant bilinear form and hence that the algebra is symmetrisable. This is not the case for the formula of Berman and Moody [13] (B.M.) which is based on Kumar-Kac-Weyl formula [12]. Coleman and Howard [14] have implemented a modified version of B.M formula on a computer. This, however, assumes that the Cartan Matrix is non-degenerate and so cannot be used for the affine algebras considered in this paper If somehow the program can be modified we have a fast method of calculating the Kostant function. Work in this direction is in progress.

ACKNOWLEDGMENT. We gratefully acknowledge several illuminating discussions with $R$. Ghosh and $R$. Balasubramaniam. The possibility of the infinite product identities playing a crucial role was first mentioned to us by $\mathrm{K}$. Ramachandra. We thank him for this. We thank the referee for informing us of the formula of Berman and Moody. We thank Brenda Rainey for typing the paper to the camera ready form.

\section{REFERENCES}

[1] TARSKI, J., Partition function for certain simple Lie algebras, J. Math. Phys. 4 (1963), 569-574.

[2] GRUBER, B and SANTHANAM, T.S., $S U(3)$ compact formula for $D\left(m^{\prime}\right) \otimes D(m)$ and for multiplicity $M^{m^{\prime}}\left(m^{\prime \prime}\right)$ of $m^{\prime \prime} \in D\left(M^{\prime}\right)$, Nuovo. cimento 45A (1966), 1046-1049.

[3] KLIMYK, A.U., Multiplicities of weights of representations and multiplicities of representations of semisimple Lie algebras, Soviet Math. Dokl. 8 (1967), 1531-1534.

[4] GRUBER, B. and WEBER, H.J., On the construction of weight diagrams for $S O(5), S p(4)$, and $G_{2}$, Proc. Roy. Irish Acad. Sect A, 66A (1968), 31-40.

[5] BELINFANTE, J.G.F., KOLMAN, B. and JACOBSON, N., Lie Algebras, InterScience Publishers, Inc., New York, 1962.

[6] RADHAKRISHNAN, D. and SANTHANAM, T.S., International multiplicity structure and Clebsch-Gordan series for the exceptional group G(2), J. Math. Phys. 8 (1967), 2206-2209 and SANTHANAM, T.S., Generating functions of classical groups and evaluations of partition functions, J. Math. Phys. 1 (1969), 1704-1710.

[7] KAC, V.G. and PETERSON, D.H., Infinite-dimensional Lie algebras, theta functions and modular forms, Adv. in Math. 53 (1984), 125-264.

[8] VIRASORO, M., Subsidiary conditions and ghosts in dual-resonance models, Phys. Rev. D1 (1970), 2933-2936.

[9] CARLITZ, L., Generating functions and partition functions, Proc. of Symp. in Pure Math., Vol. VIII, Am. Math. Soc. (1965), 144-169 and ANDREWS, G.E, The Theory of Partitions, Encyclopedia of Maths and Its Applications, Addison-Wesley, MA. (1976) 207.

[10] TANNERY, J. and MOLK, J., Elements de la Theoric des Functions Elliptiques, Paris, 1898

[11] MACDONALD, I., Affine root systems and Dedekind's $Z$-function, Invent. Math., Canad. Math. Soc. Conference Proc. 15 (1972), 91-143; see also, LOOIJENGA, E., Invariant theory for generalized root systems, Invent. Math. 611 (1980), 1-32.

[12] KAC, V.G., Infinite Dimensional Lie Algebras, Cambridge University Press, 1985.

[13] BERMAN, S and MOODY, R.V., Lie algebra multiplicites, Proc. AMS, 76 (1979), 223-228

[14] COLEMAN, A.J. and HOWARD, C.R., Root multiplicities for general Kac-Moody algebras Math. Reb. Acad. Sci. Canada, XI (1989), 15-18. 


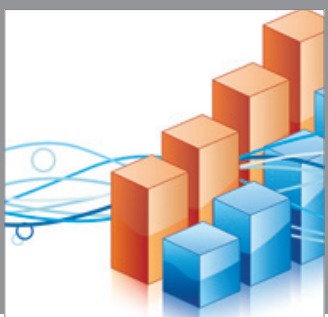

Advances in

Operations Research

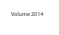

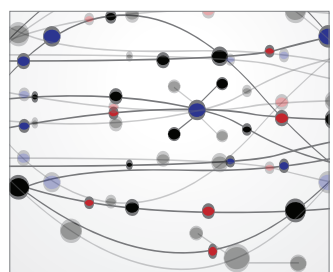

\section{The Scientific} World Journal
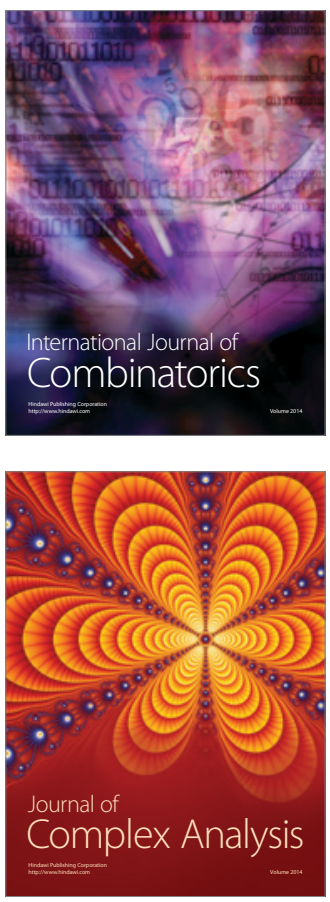

International Journal of

Mathematics and

Mathematical

Sciences
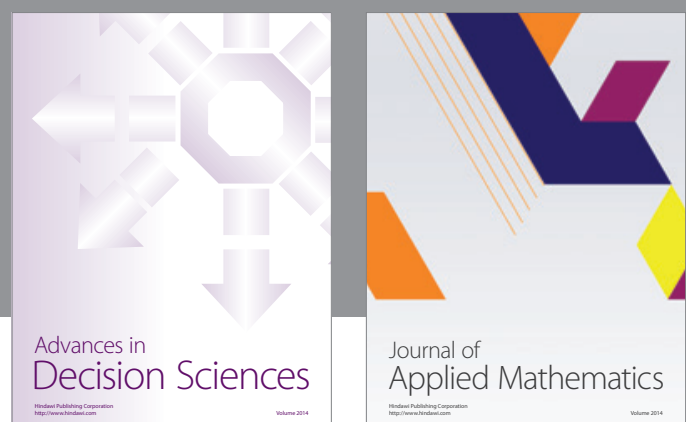

Journal of

Applied Mathematics
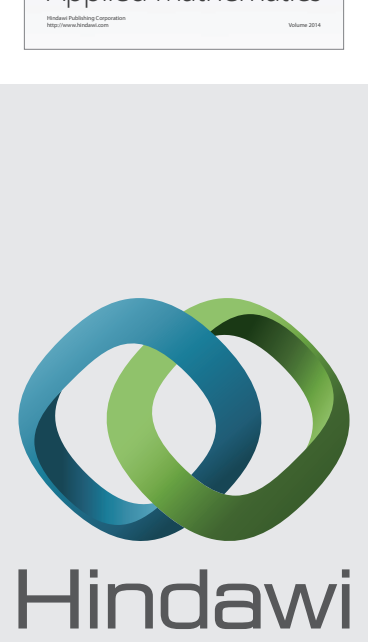

Submit your manuscripts at http://www.hindawi.com
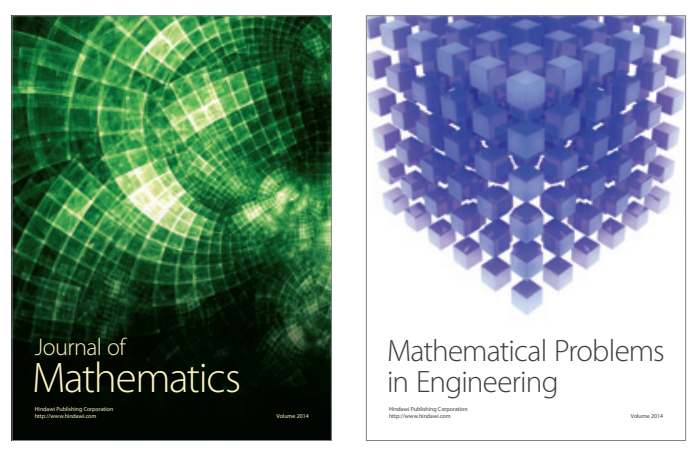

Mathematical Problems in Engineering
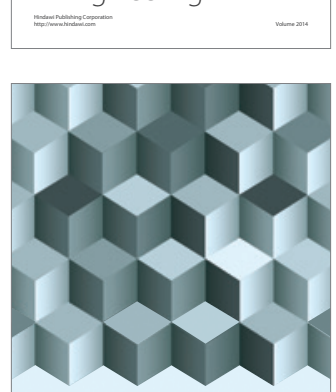

Journal of

Function Spaces
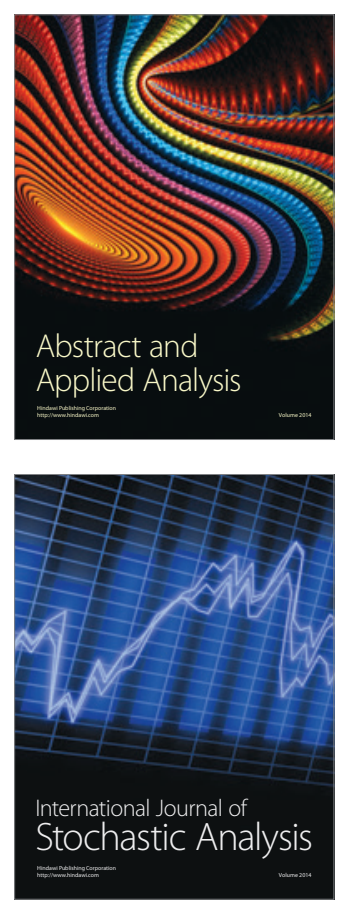

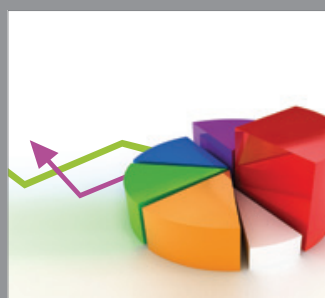

ournal of

Probability and Statistics

Promensencen
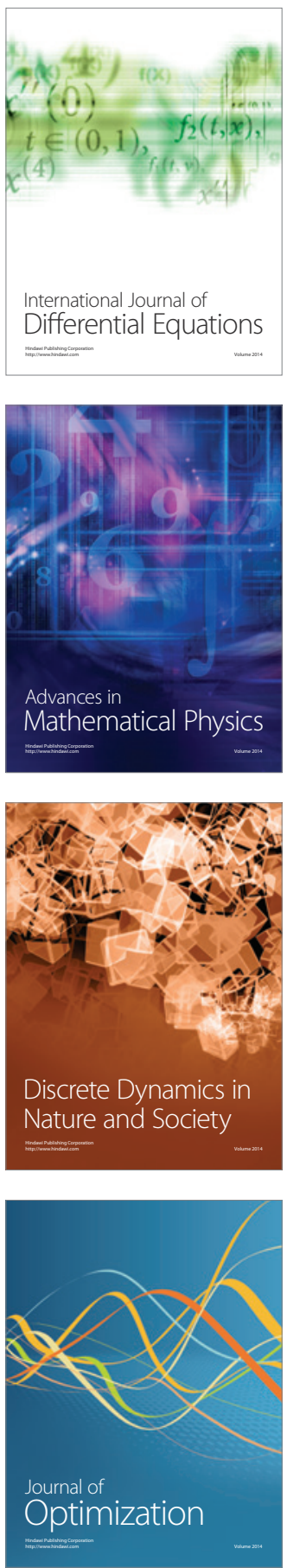